PITHA 09/32

RECAPP-HRI-2009-021

\title{
Bulk Higgs field in a Randall-Sundrum model with nonvanishing brane cosmological constant
}

\author{
Paramita Dey * \\ Institut für Theoretische Physik E, \\ RWTH Aachen, D-52056 Aachen, Germany \\ Biswarup Mukhopadhyaya ${ }^{\dagger}$ \\ Regional Center for Accelerator-based Particle Physics, \\ Harish-Chandra Research Institute, Chhatnag Road, Jhusi, Allahabad - 211 019, India \\ Soumitra SenGupta $\ddagger$ \\ Department of Theoretical Physics and Center for Theoretical Sciences, \\ Indian Association for the Cultivation of Science, Kolkata - 700 032, India
}

\begin{abstract}
We consider the possibility of Higgs mechanism in the bulk in a generalised Randall-Sundrum model, where a nonvanishing cosmological constant is induced on the visible brane. This scenario has the advantage of accommodating positive tension of the visible brane and thus ensures stability of the model. It is shown that several problems usually associated with this mechanism are avoided if some dimensionful parameters in the bulk are allowed to lie a little below the Planck mass. The most important of these is keeping the lowest massive mode in the scale of the standard electroweak model, and at the same time reducing the gauge coupling of the next excited state, thus ameliorating otherwise stringent phenomenological constraints.
\end{abstract}

\section{INTRODUCTION}

Extra dimensional models with a warped background, first proposed by Randall and Sundrum (RS) [1], offer a novel explanation of the hierarchy between the Planck $\left(M_{P}\right)$ and electroweak $(\mathrm{EW})$ energy scales. Such theories postulate that our universe is five-dimensional, described by the metric

$$
d s^{2}=e^{-2 \sigma(y)} \eta_{\mu \nu} d x^{\mu} d x^{\nu}-d y^{2},
$$

where $y=r_{c} \phi$ denotes the extra spacelike dimension compactified on $S^{1} / Z_{2}$ with radius $r_{c}, \sigma(y)=k y$, and $k \equiv \sqrt{-\Lambda / 12 M_{P}^{3}}$ denotes the curvature scale determined by the negative 5 - $\mathrm{d}$ cosmological constant $\Lambda$, and thus, is of the order of the Planck mass $\left(M_{P}\right)$. Two 3-branes are located at the boundaries at $y=0$ and $\pi r_{c}$; the latter contains the physics of the standard model (SM) of elementary particles and is called the 'visible' brane. The exponential 'warp' factor provides the aforementioned hierarchy of mass parameters, once projections on the "visible' brane are taken, for $k r_{c} \simeq 12$ [1]. Since this allows all mass parameters in the 5-d theory, including $k$ and $1 / r_{c}$ to lie in the vicinity of the Planck scale, the solution can be deemed natural.

\footnotetext{
*E-mail: paramita@physik.rwth-aachen.de

†E-mail: biswarup@mri.ernet.in

${ }^{\ddagger}$ E-mail: tpssg@iacs.res.in
}

The above set-up, though particularly successful in resolving the hierarchy issue without bringing in arbitrary intermediate energy scales, has some unsatisfactory but inevitable features;

- The tension of the visible brane, fine tuned to realise 4-d Poincare invariance, turns out to be negative [2, 3]. This makes the brane configuration intrinsically unstable.

- The visible brane, being flat, has zero cosmological constant. It is desirable to generalise the RS scenario to accommodate non-zero values of this constant.

- Though all the standard model (SM) particles were assumed to be confined to the visible brane in the original RS-theory [1], later attempts revealed that all SM fermions and gauge bosons can, in principle, propagate in the fifth dimension [4 7]. The Higgs however remains as the only exception in the sense that the hierarchy issue with a bulk Higgs field cannot be addressed unless the "minimal" set-up is extended with additional symmetries [5, 6, , 8 [1].

A recent work 2] has suggested a solution to the first of these problems by showing that one can take other solutions to the warped geometry, where positive brane tension can be achieved. This requires a negative bulk cosmological constant, as in the original RS theory [1], but also generates a non-zero cosmological constant on the visible brane [12]. The modified warp factor of this 
'generalised' RS scenario is a function of the induced cosmological constant on the brane, and tends to the original RS exponential warp factor in the limit when the brane cosmological constant goes to zero. By demanding that the numerical value of the exponent has to be the same as that in the minimal RS model, this warp factor produces the required Planck-to TeV-scale warping, such that different values of the induced brane cosmological constant correspond to different values of curvature $k$, for a given value of the brane separation scale $r_{c}$ (unlike the original RS model, where $k r_{c} \simeq 12[1]$ ).

Although the sign of the brane cosmological constant turns out be negative in the region of the parameter space corresponding to positive brane tension [2], its magnitude remains restricted to rather small values, once this scenario is used to also generate masses for neutrinos [13] via massive bulk neutrinos. The observed small positive value of the cosmological constant still necessitates some new physics on the 3-brane to cancel the induced negative cosmological constant. However, the smaller magnitude lessens the demand on such a cancellation making this scenario more favourable. The stability of the visible brane makes it even more tempting to studying particle phenomenology.

The object of this paper is to study whether this "generalised" RS scenario can accommodate a bulk Higgs field while addressing the hierarchy issue. In general, a number of difficulties in doing this have been pointed out in the literature [5, 6, 8, 9]. These include

- The bulk Higgs vev, generated through spontaneous symmetry breaking (SSB) in bulk, is of large magnitude. This, in turn, lends a large bulk mass to the gauge bosons coupling to the Higgs in the bulk. Consequently, the lowest state in the KK tower of the gauge boson on the visible brane is inadmissibly massive, failing to comply with the mass requirement of $\leq 100 \mathrm{GeV}$ in the standard electroweak theory.

- In the usual RS scenario, it has been shown that, for vanishing bulk mass of a bulk gauge boson, the first excited state in the KK tower acquires an unacceptably large coupling with matter. This puts a rather stringent restriction on the mass of such a state, in view of not only the direct search bounds at the Fermilab Tevatron but also in terms of precision electroweak constraints and limits on effective four-fermion interactions. It is possible to avoid such constraints if there is a bulk mass, as we shall show in section [V] However, there is still the need of explaining the case of the photon which cannot acquire a bulk mass, and is therefore beset with the problem related to its first massive excitation.

In this work, we suggest solutions to some of the above problems, and indicate possibilities of solving the others, in the context of the generalised RS scenario allowing a brane cosmological constant. We emphasise at the outset that, while the essence of the RS philosophy is to have bulk mass parameters only in the neighbourhood of the Planck scale, this does not rule out $k$ and $1 / r_{c}$ from being somewhat less than, but close to, this order [14]. It is thus our view that having both of the above parameters in the region $10^{16-17} \mathrm{GeV}$ is consistent with the spirit of an RS-like theory. We show that, allowing simultaneously lowered values of $k$ and $1 / r_{c}$, one obtains smaller values of the gauge boson mass on the brane, thus fitting experimental data. In addition, we are also able to show that the ratio between the gauge couplings of the lowest and first excited states on the brane is brought down to a level where direct search bounds from colliders are avoided.

In principle, this flexibility can be employed in the minimal RS model [1] itself, by lowering the values of $k$ and $1 / r_{c}$ in a correlated manner. In spite of recognising this, we use the generalised model [2] to illustrate our point, for the following reasons. First of all, this model allows a positive brane tension, thereby allowing a stable scenario. Secondly, it has been employed earlier to accommodate a scheme of neutrino mass generation [13], where specific ranges of $k$ and $1 / r_{c}$ are found to be favoured by available data. We wish to see if a solution to the problem of bulk Higgs mechanism can be found with parameters in the same range. Thirdly, some solutions to the problem of the bulk photon scenario can be thought of, for example, by proposing part of the symmetry breaking process to take place on the brane itself, by utilising terms in the Higgs Lagrangian with a $\delta$-function peaking at the visible brane. The success of such schemes often depends on the existence of a curvature-Higgs coupling term in the bulk along with a higher curvature GaussBonnet extension [10]. Such a model however encounters a stability problem [15] when the brane tension is negative. It is thus expected that the generalised RS scheme will accommodate such terms, whereby the issue of symmetry breaking can be partly transferred to the brane, thus making a massless photon (and some other features of W-and Z-interactions) realistic. We maintain that our main thrust is on suggesting the scheme of the Higgs vev in the bulk generating acceptable massive gauge bosons on the brane. Indeed, there are difficulties with various precision observables in spite of this, which may require additional new physics. However, that does not undermine the basic scheme proposed here, and the usefulness of having the chosen generalised RS scenario.

We organise our paper as follows. In Section 2, we outline the main problems of keeping the Higgs in the 5 -d bulk if one sticks to the original RS proposition. In Section 3, we briefly describe the essential features of the generalised RS scenario. The possibility of having the Higgs in the bulk in this scenario, and some numerical results of our study, are presented in section 4 . We summarise and conclude in section 5 . 


\section{PROBLEMS WITH A BULK HIGGS FIELD}

The problem of having the mechanism of spontaneous symmetry breaking in the 5 -d bulk in the original RS formalism has already been discussed in detail in several works [5, 6, 8, 9]. In this section we briefly outline the essential points by assuming that the gauge bosons and the Higgs are bulk fields. The fermions can either be bulk fields, or brane-localised.

If the Higgs boson $(H)$ is assumed to be a bulk field, the corresponding 5 -d potential takes the form,

$$
V(H)=-\mu^{2} H^{\dagger} H+\frac{\lambda_{5 d}}{2}\left(H^{\dagger} H\right)^{2}
$$

where $\mu>0$, and the Higgs field develops a vacuum expectation value (vev) in the bulk $\sim \sqrt{\mu^{2} / \lambda_{5 d}} . H$ is weakly coupled when $\langle H\rangle$, its vev, is of the same order as $\mu$. This vev will generate a bulk mass term $M$ for the bulk gauge boson $A_{s}\left[\equiv A_{\rho}, A_{5}\right]\left(x_{\mu}, \phi\right)$, which, being a 5d parameter, should be of the order of Planck scale to be consistent with the basic spirit of the RS scenario [1]. Through a process similar to that in $4-\mathrm{d}, M \sim<H>$.

Starting from the 5-d Lagrangian for the gauge fields in the RS background and making use of $Z_{2}$ orbifold conditions $\partial_{5} A_{\mu}\left(x_{\mu}, \phi=\phi_{i}\right)=0=A_{5}\left(x_{\mu}, \phi=\phi_{i}\right)$ on the boundaries $\phi_{i}$, one can arrive at the equation,

$$
\partial^{\mu}\left(\partial_{\mu} A_{\rho}^{a}\right)-\frac{1}{r_{c}^{2}} \frac{\partial}{\partial \phi}\left(e^{-2 \sigma} \frac{\partial}{\partial \phi} A_{\rho}^{a}\right)+M^{2} e^{-2 \sigma} A_{\rho}^{a}=0
$$

in the $A_{5}^{a}=0$ gauge, $a$ being the gauge index. The 5 -d field $A_{\rho}^{a}$ can be expanded into 4 -d KK modes as,

$$
A_{\rho}^{a}\left(x_{\mu}, \phi\right)=\sum_{n} A_{\rho}^{a(n)}\left(x_{\mu}\right) \frac{f_{n}(\phi)}{\sqrt{r_{c}}}
$$

where each KK mode satisfies $\left(\square+m_{n}^{2}\right) A_{\rho}^{a(n)}=0, m_{n}$ being the corresponding KK mass.

It should be noted that equation (2.2) is valid for both Abelian and non-Abelian gauge fields that acquire mass through the vev of $H$ in 5 -dimensions. This is because each component of $A_{\mu}^{a}$ still satisfies $\left(\square+m_{n}^{2}\right) A_{\rho}^{a(n)}=0$ [16], when one considers the 'free' part of its lagrangian, leaving out the self-interactions, a procedure that is routine when one expands the field in normal modes. Thus the solutions of (2.2) should be the starting point in the KK decomposition of non-Abelian gauge fields as well.

Using this expansion in Eq.(2.2) we get the relation satisfied by $f_{n}$ as

$$
-\frac{1}{r_{c}^{2}} \frac{\partial}{\partial \phi}\left(e^{-2 \sigma} \frac{\partial}{\partial \phi} f_{n}\right)+M^{2} e^{-2 \sigma} f_{n}=m_{n}^{2} f_{n}
$$

The transformations $f_{n}=e^{\sigma} \chi_{n}$ and $Z_{n}=e^{\sigma} m_{n} / k \bmod -$ ify the above equation into the much known form

$$
Z_{n}^{2} \frac{d^{2} \chi_{n}}{d Z_{n}^{2}}+Z_{n} \frac{d \chi_{n}}{d Z_{n}}+\left[Z_{n}^{2}-\left(1+\frac{M^{2}}{k^{2}}\right)\right] \chi_{n}=0
$$

\begin{tabular}{|c|c|c|c|c|c|c|c|c|}
\hline & \multicolumn{2}{|c|}{$M=0$} & \multicolumn{2}{c|}{$M=0.1 k$} & \multicolumn{2}{c|}{$M=0.5 k$} & \multicolumn{2}{c|}{$M=k$} \\
\hline$n$ & $x_{n}$ & $m_{n}$ & $x_{n}$ & $m_{n}$ & $x_{n}$ & $m_{n}$ & $x_{n}$ & $m_{n}$ \\
\hline 0 & 0 & 0 & 2.41 & 2.41 & 2.54 & 2.54 & 2.87 & 2.87 \\
1 & 2.40 & 2.40 & 5.53 & 5.53 & 5.68 & 5.68 & 6.09 & 6.09 \\
2 & 5.52 & 5.52 & 8.66 & 8.66 & 8.82 & 8.82 & 9.25 & 9.25 \\
3 & 8.65 & 8.65 & 11.80 & 11.80 & 11.97 & 11.97 & 12.40 & 12.40 \\
\hline
\end{tabular}

TABLE I: Solutions of the eigenvalue equation for obtaining masses of $K K$ gauge bosons in the $R S$-model for different ratios of $M / k$ are listed. For $k \sim M_{P}$, the corresponding masses are given in $\mathrm{Te} V$.

the solutions of which are the Bessel functions $J_{\alpha}$ of order $\alpha=\sqrt{\left(1+M^{2} / k^{2}\right)}$. Thus one arrives at

$$
f_{n}=\frac{e^{\sigma}}{N_{n}} J_{\alpha}\left(\frac{m_{n}}{k} e^{\sigma}\right)
$$

where $N_{n}$ normalises $f_{n}$. The corresponding mass eigenvalues $m_{n}$ can then be found by solving the eigenvalue equation,

$$
\frac{x_{n}}{2} J_{\alpha-1}\left(x_{n}\right)+J_{\alpha}\left(x_{n}\right)-\frac{x_{n}}{2} J_{\alpha+1}\left(x_{n}\right)=0,
$$

which is obtained from the continuity of $d f_{n} / d \phi$ on the visible brane $(\phi=\pi)$. Here

$$
x_{n}=m_{n} e^{\sigma(\pi)} / k
$$

The smallest root $x_{0}$ of the eigenvalue equation yields the lowest mass eigenvalue $m_{0}$ and, the corresponding KK mode $A_{\rho}^{(0)}\left(x_{\mu}\right)$ (the so called "zeroth" KK state) is interpreted as the standard model (SM) field. If we solve Eq.(2.7) for $M=0$, the lowest eigenvalue turns out to be $m_{0}=0$ (see Table I). Following the previous argument therefore, we know that this has to be the case for massless SM gauge bosons like photon [6, 8]. Similarly, for the massive ones like $W$ or $Z$, we should start with a nonzero $M\left(\sim M_{P}\right)$, and expect $m_{0} \simeq 100 \mathrm{GeV}$. However, when we actually solve Eq.(2.7) for this case, the lowest non-trivial solution is obtained at $x_{0} \sim 2$ for $M \sim k$ (note, from Eq.(2.4), that the zero-eigenvalue solution demands the corresponding wave-function to vanish altogether and is thus a trivial solution). In Table I we list the exact values of $x_{0}$ corresponding to different nonzero ratios of $M / k$. Recall that $e^{\sigma(\pi)}$ must be $\sim 10^{16}$ in order to address the gauge hierarchy issue. As a result, from Eq.(2.8) the lowest eigenvalue for this case turns out to be $\sim 2 \mathrm{TeV}$ (see Table I). Naturally therefore, the corresponding zeroth KK state in this case cannot be interpreted as any of the known massive SM gauge bosons as such. Such an interpretation will be possible if we could somehow lower the value of $m_{0}$ to the required mass regime, which, in turn, can be achieved if only we allow $M / k<<1$. This should be obvious from the fact that the limit $M \rightarrow 0$ must reproduce $m_{0}=0$. In fact, it has been shown in [8] that to have $m_{0}$ in the $100 \mathrm{GeV}$ 
regime, we must have $M / k$ even lower than $\sim 10^{-10}$. This however is undesirable, since this destroys the main spirit of the RS scenario by bringing in a bulk mass parameter much smaller than the Planck mass.

It is, however, obvious from Eq.(2.8), that the above problem of having the mass of the zeroth KK gauge boson in the required regime can be addressed if one is allowed to lower the value of $k$ below the Planck scale. But, the original formalism of RS does not allow such a freedom of $k$, since, to address the hierarchy issue one must keep $k r_{c} \sim 12$ [1]. This is exactly where the generalised RS scenario may turn out to be particularly useful. As mentioned before, the required warping for this scenario does allow different values of curvature $k$, for a given value of the brane separation scale $r_{c}$ and the induced cosmological constant on the visible brane [2]. Thus, it provides a chance to tune the values of the gauge field mass parameters. Furthermore it may be possible that those relevant $k$ values correspond to positive tension for the visible brane. In such a situation therefore one can successfully achieve a five dimensional spontaneous symmetry breaking mechanism generating masses for SM fields on a stable visible brane with non zero brane cosmological constant. To this end we probe the generalised RS scenario in the next two sections.

\section{RS SCENARIO WITH A GENERALISED WARP FACTOR}

The details of a generalised RS scenario with a nonzero brane cosmological constant has been discussed in detail in [2, 17, 18]. In this section we briefly talk about the essential features of this scenario.

Instead of the metric of Eq.11.1 suppose we use one with a more general warp factor $A(y)$,

$$
d s^{2}=e^{-2 A(y)} \eta_{\mu \nu} d x^{\mu} d x^{\nu}-d y^{2}
$$

and evaluate $A(y)$ by extremising the action. As [2] shows, starting with an anti-de Sitter (AdS) bulk $(\Lambda<$ 0 ), it is possible to have a constant curvature brane spacetime which can be either $\operatorname{AdS}(\Omega<0)$ or $\mathrm{dS}(\Omega>0)$ where $\Omega$ is the induced cosmological constant on the visible brane.

For $\Omega<0$, the solution for the warp factor turns out to be

$$
e^{-A(y)}=\omega \cosh \left(\ln \frac{\omega}{1+\sqrt{1-\omega^{2}}}+k y\right)
$$

where $\omega^{2}=-\Omega^{2} / k^{2}$ is the absolute value of the dimensionless quantity obtained out of $\Omega$. Note that the original RS solution $A=k y$ is recovered in the limit $\omega^{2} \rightarrow 0$. If we set $e^{-A} \simeq 10^{-16}$ to ensure the hierarchy between the Planck and EW scales, we find two solutions for $k r_{c} \pi$ for every $\omega^{2}$, corresponding to positive and negative brane tension, respectively. No solution, however, exists for $\omega^{2}>10^{-32}$. These solutions can then be used to determine the corresponding visible brane tensions using the equation [2]:

$$
\mathcal{V}_{\text {vis }}=12 M^{3} k\left[\frac{\frac{\omega^{2}}{c_{1}^{2}} e^{2 k r_{c} \pi}-1}{\frac{\omega^{2}}{c_{1}^{2}} e^{2 k r_{c} \pi}+1}\right]
$$

Recall that we are interested in having a positive tension for the visible brane in order to have stable brane configuration. One of the solutions, which yields the usual $\mathrm{RS}$ value of $k r_{c} \pi \simeq 36.84$ in the limit of near-vanishing $\omega$, always corresponds to a negative brane tension, and hence is not relevant to our cause. The other solution, on the other hand, leads to positive brane tension and gives increasing values of $k r_{c} \pi$ as $\omega^{2}$ decreases, leading to $k r_{c} \pi \simeq 250.07$ for $\omega^{2} \rightarrow 10^{-124}$ (see Figures 1 and 2 in reference [2]). On the whole, a rather wide region in the $\omega^{2}-k r_{c} \pi$ space is generally allowed, a large part of which yields positive brane tension (region III in Figure 1 in reference [2]). For $\Omega>0$, it is not possible to have positive tension for the visible brane, and hence, we exclude this case from further discussions.

Thus, the region of our interest in the $\omega^{2}-k r_{c} \pi$ has a negative brane cosmological constant, which is a necessary condition for positive brane tension [2, 17, 18]. While the requirement of a positive tension brane can lead to values of $\omega^{2}$ as high as $10^{-32}$ (see Figure 1 in reference [2]), it may be desirable to keep $\omega^{2}$ as small as possible. This way one can demand much less cancellation from some hitherto unknown physics to play a role in its observed small positive value. Interestingly, if a mechanism of neutrino mass generation is envisioned in terms of bulk sterile neutrinos [6, 7, 19, 20] in such a generalised warped geometry, much smaller values of $\omega^{2}$ than $10^{-32}$ can be shown to be consistent with phenomenology [13].

\section{BULK HIGGS IN THE GENERALISED RS SCENARIO}

If the Higgs is a bulk field in the generalised RS scenario described in section III, then proceeding just as in section II] the equation of motion for the $n^{\text {th }}$ KK mode $\left(f_{n}\right)$ of mass $m_{n}$ of a bulk gauge field is obtained as,

$$
\begin{gathered}
Z_{n}^{2} \frac{d^{2} \chi_{n}}{d Z_{n}^{2}}+Z_{n} \frac{d \chi_{n}}{d Z_{n}}+\left[Z_{n}^{2}-\left(1+\frac{M^{2}}{k^{2}}\right)\right] \chi_{n}= \\
\operatorname{cosech}^{2}(B)\left(Z_{n} \frac{d \chi_{n}}{d Z_{n}}-\left[Z_{n}^{2}-\left(1+\frac{M^{2}}{k^{2}}\right)\right] \chi_{n}\right)
\end{gathered}
$$

where $Z_{n}=\frac{m_{n}}{k} e^{A}, \chi_{n}=e^{-A} f_{n}, c_{1}=1+\sqrt{1-\omega^{2}}$ and $\operatorname{cosech}^{2}(B)=\operatorname{cosech}^{2}\left(\ln \left(\omega / c_{1}\right)+k r_{c} \phi\right)$. It can be easily checked from Eq. (3.2) that the terms on the right hand side of the above equation are proportional to $\omega^{2}$ or its higher powers. Thus they vanish in the limit $\omega \rightarrow 0$, and as expected, we get back the corresponding equation for the original RS model [1]. In fact, as mentioned previously we will also be interested in as small values of $\omega$ as 


\begin{tabular}{|c|c|c|c|c|c|c|c|c|}
\hline & \multicolumn{2}{|c|}{$M=0$} & \multicolumn{2}{c|}{$M=0.1 k$} & \multicolumn{2}{c|}{$M=0.5 k$} & \multicolumn{2}{c|}{$M=k$} \\
\hline$n$ & $x_{n}$ & $m_{n}$ & $x_{n}$ & $m_{n}$ & $x_{n}$ & $m_{n}$ & $x_{n}$ & $m_{n}$ \\
\hline 0 & 0 & 0 & 2.41 & 96.40 & 2.54 & 101.60 & 2.87 & 114.80 \\
1 & 2.40 & 96.00 & 5.53 & 221.20 & 5.68 & 227.20 & 6.09 & 243.6 \\
2 & 5.52 & 220.80 & 8.66 & 346.40 & 8.82 & 352.8 & 9.25 & 370.00 \\
3 & 8.65 & 346.00 & 11.80 & 472.00 & 11.97 & 478.80 & 12.40 & 496.00 \\
\hline
\end{tabular}

TABLE II: Solutions of the eigenvalue equation for obtaining masses of KK gauge bosons in the generalised $R S$-model for different ratios of $M / k$ are listed. Note that the solutions are exactly same as those obtained for the usual RS-theory (listed in Table I). For $k \sim 4 \times 10^{17} \mathrm{GeV}$, the corresponding masses are given in $\mathrm{GeV}$.

possible [13], so that we can drop the right hand side of Eq.(4.1) for all practical purposes. Thus, the solutions of $\chi_{n}$ from Eq.(4.1) will still be the Bessel functions $J_{\alpha}\left(Z_{n}\right)$, and the eigenvalues will, as usual, be obtained from the continuity of $f_{n}$ at $\phi=\pi$,

$$
\frac{x_{n}}{2} J_{\alpha-1}\left(x_{n}\right)+J_{\alpha}\left(x_{n}\right)-\frac{x_{n}}{2} J_{\alpha+1}\left(x_{n}\right)=0
$$

with $x_{n}$ now modified as [18]

$$
x_{n}=m_{n} e^{A\left(r_{c} \pi\right)} / k
$$

and $\alpha=\sqrt{\left(1+M^{2} / k^{2}\right)}$ as before. This clearly means that the solutions $x_{n}$ for different $n$ as listed in Table I (for $M \neq 0$ ) are still valid (also listed in Table Џ). Therefore, $x_{0} \sim 2$ just as before, and we will have to use $e^{A\left(r_{c} \pi\right)} \sim 10^{16}$ while evaluating the eigen value $m_{0}$. However, since in this case we can indeed allow a somewhat lower value for $k$, it is possible to have $m_{0}$ in the $100 \mathrm{GeV}$ regime; for example, $k \sim 4 \times 10^{17} \mathrm{GeV}$ yields $m_{0} \sim 96$ $\mathrm{GeV}$. For this choice of $k$, if, for example, for $\omega^{2}$ lying in the range $\sim 10^{-80}-10^{-50}, 1 / r_{c}$ occurs around $10^{16} \mathrm{GeV}$ (see equation 21 in [2]). This allows us to simultaneously satisfy the neutrino data [13]. It is also clear from these figures that for smaller values of $\omega^{2}$, the separation of the scales of $k$ and $1 / r_{c}$ increases. In any case, the zeroth KK mode in this scenario can indeed be identified with known SM gauge bosons.

However, note from Eq. (4.3), that such a lowering of the value of $k$ not only lowers the mass of the zeroth KK state, it lowers the masses of all the higher KK modes also. As a result, in this generalised RS model with $k \sim$ $4 \times 10^{17}$ the first KK excitation corresponding to $x_{1} \sim$ 5.5 has a mass $m_{1} \sim 220 \mathrm{GeV}$ (see Table II). Recall that in the original RS-theory with $K \sim M_{P}$, the same state had a mass $m_{1} \sim 5.5 \mathrm{TeV}$ (see Table 【). In a similar way, the mass of the second KK state will now be $m_{2} \sim 350 \mathrm{GeV}$ instead of $9 \mathrm{TeV}$ in the original RSmodel, and so on. Whether or not these KK-states with such low masses can survive the experimental constraints from direct observations at the Tevatron [21] is decided by the strengths of their corresponding couplings to SM fermions.
To estimate the strengths of such couplings, let us first assume that the fermions are also bulk fields in this generalised RS scenario. We refer the reader to Ref. [17] for a treatment of such fermions. The 5-d Lagrangian describing the gauge interaction of the bulk fermions with the bulk gauge field is then given as [8]

$$
e^{-1} \mathcal{L}=g_{5 d} \bar{\Psi}\left(x^{\mu}, \phi\right) i \Gamma \underline{S}_{\underline{S}}^{S}(\phi) A_{S}\left(x^{\mu}, \phi\right) \Psi\left(x^{\mu}, \phi\right)
$$

Using the expansions of both types of bulk fields in terms of KK excitations in the above equation and integrating over the extra dimension, the expression for the coupling of the $n^{\text {th }}$ KK gauge boson to the massless zero-mode fermion (i.e. SM fermion) bilinear is obtained as,

$$
g_{n} \sim g_{5 d} \frac{\sqrt{2 k}}{N_{n}\left(B_{c}-1\right)} \frac{k^{2}}{m_{n}^{2}} \int_{0}^{1} x d x\left[J_{\alpha}(x)\right]
$$

where $B_{c}=e^{A\left(r_{c} \pi\right)}$. Numerical evaluation of the above integration yields $g_{1} / g_{0} \sim 0.134, g_{2} / g_{0} \sim 0.132$ and $g_{n} / g_{0}<<1$ for higher $n$. As a result of such reduced couplings, the Tevatron limit of $M_{T}>700 \mathrm{GeV}$ on the mass of a heavy vector boson [21] (for a coupling of the same strength as in the $\mathrm{SM}$ ) is reduced to $M_{T}>95 \mathrm{GeV}$ $(\sim 700 \times 0.134)$. Thus, the KK gauge bosons, although have somewhat lower masses for a low value of $k$, still survive the experimental constraints.

Note that the ratios of the strengths of $g_{n}$ obtained in our case have an interesting correspondence to those in section IV of Ref. [8] which deals with bulk gauge fields of zero bulk mass. Denoting their couplings by $\tilde{g}_{n}$, the correspondence can be described as follows. The ratio $g_{1} / g_{0}$ in our case is $\sim \tilde{g}_{2} / \tilde{g}_{1}$ in their case, and so on. In other words, the SM gauge coupling $g_{0}$ in our case corresponds to the first KK gauge coupling $\tilde{g}_{1}$ in their case, and so on. Such a correspondence is easy to understand from the $x_{n}$ values listed in Table $\llbracket$ or for both $M=0$ and $M \neq 0$. Note that $x_{0}$ for $M \neq 0$ is $\sim x_{1}$ for $M=0, x_{1}$ for $M \neq 0$ is $\sim x_{2}$ for $M=0$ and the like. This correspondence can then be translated into a similar correspondence among the wavefunctions $f_{n}$ which are functions of $x_{n}$, and finally into the couplings $\left(g_{n}\right.$ or $\left.\tilde{g}_{n}\right)$ which are decided by these wavefunctions. Naturally, the coupling $\tilde{g}_{0}$ in Ref. [8] (i.e. the SM coupling in their case) has no analogue in our case, since there is no zero-mass gauge boson for $M \neq 0$.

It has been already mentioned that the next-excited state for any spin-1 KK tower generally tends to have enhanced coupling, which may not only cause conflicts with direct search bounds but also violate the limits imposed by precision electroweak observables. For a gauge boson which has acquired a bulk mass through SSB in five-dimensions, this problem seems to be avoided, since this time one considers the second massive mode in the tower vis-a-vis the first one, where the former has relatively suppressed coupling with matter [8]. However, the problem may still persist for the KK tower of the five-dimensional photon state which has no bulk mass. Furthermore, one may encounter difficulties in complying with the observed relations between the $\mathrm{W}$-and Zmasses, or their relative gauge couplings [5, 8, [9]. As 
already suggested, a number of remedies for this problem may be considered. These include (a) some contact interaction on the brane playing a role in the SSB of the $\mathrm{U}(1)$ symmetry, (b) a separate brane kinetic energy term for the U(1) part of the SM [22], and (c) some altogether new physics (additional fields) which may cancel the contributions to the precision electroweak variables.

Another interesting solution to the problem of enhanced coupling of the first excited state of the photon can be envisioned as follows. If one recalls Eq. (4.5) for the coupling of the first excited state, it becomes, on simplification,

$$
g_{1} \sim g \frac{\sqrt{2 \pi k r_{c}} k^{2} e^{-2 A\left(r_{c} \pi\right)}}{m_{1}^{2}}
$$

In the generalised RS model the warp factor $A\left(r_{c} \pi\right)$ for $\omega^{2}<<1$ is given (from equation 3.2) as

$$
e^{-A\left(r_{c} \pi\right)}=e^{k r_{c} \pi}\left(\omega^{2} / 4\right)+e^{-k r_{c} \pi}
$$

where the first term on the right-hand side dominates for values of $k r_{c}$ corresponding to positive brane tension.

Unlike the standard RS model where a particular value of $k r_{c}$ uniquely determines the extent of warping on the SM brane [1], here we have two-parameter solutions for the warp factor in terms of $\omega^{2}$ and $k r_{c}[2]$. The two possible values of $k r_{c}$ for any given combination of $\omega$ and $e^{A\left(r_{c} \pi\right)}$ correspond respectively to the positive and negative tension branes. If we wish to focus on the branch of solution for which both the brane tensions are positive (for the stability consideration) then the value of $k r_{c} \pi$ must be greater than 37.5 [2] i.e somewhat higher than the usual RS value $\sim 36[1]$.

In the region of solutions for $k r_{c}$ for which the brane tension is positive we obtain from Eqs.(4.6) and (4.7),

$$
g_{1} \sim g \frac{\sqrt{2 \pi k r_{c}} k^{2}\left(e^{k r_{c} \pi}\left(\omega^{2} / 4\right)\right)^{2}}{m_{1}^{2}}
$$

In order to resolve the gauge hierarchy problem, the value of the warp factor must be $10^{-16}$. This immediately yields different values of $k r_{c}$ for different choices of the brane cosmological constant $\omega^{2}$ from equation 4.7. If we now allow the value of the warp factor to be $10^{-17}$ by appropriately choosing the value of the cosmological constant $\omega^{2}$ one order lower than the value required to make the warp factor $10^{-16}$, the coupling constant for the first excited mode gets suppressed by one order (see Eq.(4.8)). Thus at the expense of changing the warp factor by just one order, we can remove the problem of unacceptable enhancement of the coupling of the first KK mode of photon when the SSB takes place in the bulk. Of course, in order to achieve acceptable values of $m_{n}$ in Eq.(4.7), one needs to have values of $k$ slightly different from that given in Table II In fact in this scheme the coupling of the first excited mode of the massive vector boson also receives an additional suppression by an order (see equation 4.5). Thus in the generalised RS model [2] the freedom of the choice of the brane cosmological constant and the corresponding $k r_{c}$ provides us an alternative path to resolve this long-standing problem of strongly coupled first excited mode for massless gauge field in the bulk. This cannot be construed as re-introducing the hierarchy problem, since an order of uncertainty in the factor connecting the Planck and electroweak scales may arise due to various kinds of unknown physics in the huge intervening range.

Before we end this section, it may be useful to comment on the issue of fermion mass generation coming from five dimensional spontaneous symmetry breaking with a bulk Higgs field. If the fermions $(F)$ are brane localised, the five dimensional Yukawa action will be given by,

$$
S_{Y}^{5}=\int d^{4} x \int d y\left[Y^{5} \bar{F} F H \delta\left(y-\pi r_{c}\right)\right]
$$

where $Y^{5}$ is the five dimensional Yukawa coupling. When the symmetry breaking takes place, the Higgs field should be replaced by $H=\langle H\rangle+h$, where $\langle H\rangle$ is the vev developed in the bulk $\left(\sim \sqrt{\mu^{2} / \lambda_{5 d}} \sim\right.$ Planck scale $)$. The insertion of $\langle H\rangle$ in the above equation generates masses for the fermions. The warp factor in RS coming through the Jacobian leads to the suppression of the large vev $\langle H\rangle$ into a mass in the electroweak range [1]. The observed spectrum of fermion masses, of course, requires widespread values of $Y^{5}$, for which there is no explanation as in SM. For the bulk fermion case, too, there exists a well-defined prescription. In this case, all the KK fermions have two different sources of mass, one being the "Yukawa mass" coming from SSB in the bulk, and the other being the usual KK mass. The complete mass matrix for this case is very similar to that described in 23], although the scenario itself is not the same as ours. The model in 23] has bulk fermions and a brane localised Higgs field, while in our case all are bulk fields. This only results in some non-zero non-diagonal entries in the mass matrix which were zero in 23] (for example, the $(3,2)$ element of the matrix in equation 4.28 of that paper).

Of course, when the fermions and the Higgs are both in the bulk, their overlap can in general be large, thus making it difficult to explain small fermion masses. This difficulty can be ameliorated by either imposing some symmetry which makes the bulk Higgs profile peak near the visible brane, or postulating a very small coefficient for the Higgs-fermion coupling in the bulk. These are of course, tentative proposals and are open to further investigations.

\section{SUMMARY AND CONCLUSIONS}

We have studied bulk Higgs mechanism in an RS scenario where a cosmological constant is induced in the brane. Such a scenario, as has been argued earlier, ensures positive tension for both of the branes located at the orbifold fixed points. Some mass parameters such as the induced bulk mass for vector bosons, the curvature 
factor $k$ (related to the bulk cosmological constant) and the inverse of the radius of the compact dimension are allowed to lie up to two orders of magnitude below the Planck mass, a practice which, we argue, does not go against the overall philosophy of RS-like theories.

We show that, once this is done, the KK tower of vector boson masses on the visible brane becomes phenomenologically acceptable. First, the lowest-lying member of the tower can have a mass within $100 \mathrm{GeV}$, thus raising hopes of answering to features of the standard electroweak model. In addition, the next excited state now is shown to have considerably reduced coupling to the standard fermions, thus avoiding phenomenological constraints which would have otherwise rendered the scenario unacceptable. The enhanced flexibility of varying $k$ and $1 / r_{c}$ enlarges the model parameter space consistent with data. Needless to say, this is feasible in this scenario because of the non-zero cosmological constant induced on the brane. Finally, we show that a somewhat less rigid allowed range for the warp factor reduces the gauge coupling of the first excited mode of the bulk photon, thereby allowing it to have masses on a low scale.

\section{ACKNOWLEDGMENT}

PD is supported through the Gottfried Wilhelm Leibniz program by the Deutsche Forschungsgemeinschaft (DFG). The work of BM was partially supported by funding available from the Department of Atomic Energy, Government of India, for the Regional Center for Accelerator-based Particle Physics (RECAPP), HarishChandra Research Institute. SSG acknowledges the hospitality of the Regional Centre for Accelerator-based Particle Physics, Harish-Chandra Research Institute, where part of the work was done.
[1] L. Randall and R. Sundrum, Phys. Rev. Lett. 83, 3370 (1999); ibid 83, 4690 (1999).

[2] S. Das, D. Maity and S. Sengupta, JHEP 05, 042 (2008).

[3] C. Csaki et al., Nucl. Phys. B584, 359 (2000); J. W. Chen, M. A. Luty and E. Ponton, JHEP, 09, 012 (2000); M. Sasaki, T. Shiromizu and K.i. Maeda, Phys. Rev. D62, 024008 (2000); J. M. Cline et al., JHEP, 06, 048 (2003); I. Navarro, JCAP, 09, 004 (2003); J. Garriga and M. Porrati, JHEP, 08, 028 (2004); H. P. Nilles, A. Papazoglou and G. Tasinato, Nucl. Phys. B677, 405 (2004); J. Vinet and J. M. Cline, Phys. Rev. D70, 083514 (2004); C.P. Burgess et al., JHEP, 11, 069 (2004); Y. Aghababaie et al., Nucl. Phys. B680, 389 (2004).

[4] W.D. Goldberger and M.B. Wise, Phys. Rev. D60, 107505 (1999).

[5] H. Davoudiasl, J.L. Hewett and T.G. Rizzo, Phys. Lett. B473 (2000) 43; H. Davoudiasl, J. L. Hewett and T. G. Rizzo, Phys. Rev. D63 075004 (2001).

[6] A. Pomarol, Phys. Lett. B486 (2000) 153.

[7] T. Gherghetta and A. Pomarol, Nucl. Phys. B586, 141 (2000); T. Gherghetta, Phys. Rev. Lett. 92161601 (2004); G. Moreau and J. I. Silva-Marcos, JHEP 03, 090 (2006).

[8] S. Chang, J. Hisano, H. Nakano, N. Okada and M. Yamaguchi, Phys. Rev. D62, 084025 (2000).

[9] C.H. Chang and J.N. Ng, Phys. Lett. B488, 390 (2000); S.J. Huber and Q. Shafi, Phys. Rev. D63, 045010 (2001).

[10] H. Davoudiasl, J.L. Hewett, B. Lillie and T.G. Rizzo, Phys. Rev. D70, 015006 (2004); H. Davoudiasl, B. Lillie and T.G. Rizzo, JHEP 0608042 (2006).

[11] K. Agashe, A. Delgado, M.J. May and R. Sundrum, JHEP 0308, 050 (2003); Y. Hosotani and M. Mabe, Phys. Lett.B615, 257 (2005); Y. Hosotani, S. Noda, Y. Sakamura and S. Shimasaki, Phys. Rev. D73, 096006 (2006); M.S. Carena, E. Ponton, J. Santiago and C.E.M. Wagner, Nucl. Phys. B759, 202 (2006); A.D. Medina, N.R. Shah and C.E.M. Wagner, Phys. Rev. D76, 095010 (2007); A. Djouadi and G. Moreau, Phys. Lett. B660,
67 (2008); M. Bauer, arXiv:0910.4876 [hep-ph]; M.E. Albrecht, M. Blanke, A.J. Buras, B. Duling and K. Gemmler, JHEP 0909, 064 (2009).

[12] J.M. Cline, C. Grojean and G. Servant, Phys. Rev. Lett. 83, 4245 (1999); P. Binetruy, C. Deffayet, V. Ellwanger and D. Langolois, Phys. Lett. B477, 285 (2000); T. Shiromizu, K. Maeda, M. Sasaki, Phys. Rev D62, 024012 (2000).

[13] P. Dey, B. Mukhopadhyaya and S. SenGupta, Phys. Rev. D80 055029 (2009).

[14] W.D. Goldberger and M.B. Wise, Phys. Rev. Lett. 83, 4922 (1999); C. Csaki, M. Graesser, L. Randall and J. Terning, Phys. Rev. D62, 045015 (2000); J. Lesgourgues, S. Pastor, M. Peloso and L. Sorbo, Phys. Lett. B489, 411 (2000).

[15] C. Charmousis and J.F. Dufaux, Phys. Rev. D70, 106002 (2004).

[16] K. Hagiwara et al., Nucl. Phys. B292, 253 (1987).

[17] R. Koley, J. Mitra and S. SenGupta, Phys. Rev. D79, 041902 (2009).

[18] J. Mitra and S. SenGupta, arXiv:0905.3984 [hep-th].

[19] Y. Grossman and M. Neubert Phys.Lett B474, 361 (2000).

[20] S. Chang et al., Phys. Rev D62, 084025 (2000); B. Bajc and G. Gabadadze, Phys. Lett. B474, 282 (2000); I. Oda, Phys. Lett. B496, 113 (2000); Jean-Luc Lehners, P. Smyth and K.S. Stelle, Nucl. Phys.B790, 89 (2008).

[21] C. Amsler et al. (Particle Data Group), Phys. Lett. B667, 1 (2008); T. Altonen et al., Phys. Rev. D78, 012008 (2008); T. Altonen et al., Phys. Rev. Lett. 102, 031801 (2009).

[22] M.S. Carena, E. Ponton, T.M.P. Tait and C.E.M. Wagner, Phys. Rev. D67, 096006 (2003); H. Davoudiasl, J.L. Hewett and T.G. Rizzo, Phys. Rev. D68 (2003) 045002.

[23] See for example, equation 4.28 of S.J. Huber, Nucl. Phys. B666, 269 (2003) arXiv:hep-ph/0303183. 